SCIREA Journal of Clinical Medicine

ISSN: 2706-8870

http://www.scirea.org/journal/CM

September 28, 2021

SCIREA

Volume 6, Issue 5, October 2021

\title{
Characteristics of cardiopulmonary ultrasonography in patients with reduced postoperative oxygenation index in PACU
}

CHAI Mei ${ }^{1}$, SU Yang ${ }^{1}$, XIONG Ying ${ }^{1}$, LUO Wei ${ }^{1}$, LIU Jie ${ }^{1}$, ZHOU Feng ${ }^{1}$, ZHANG Yanjun $^{1}$, ZHENG Xin ${ }^{1}$, ZHAO Linyan ${ }^{2}$

${ }^{1}$ Department of Anesthesiology, The Second Affiliated Hospital of Dalian Medical University, China;

${ }^{2}$ Department of Intensive Care, The Second Affiliated Hospital of Dalian Medical University, China;

Corresponding author: Zheng Xin, male, 40 years old, associate chief physician, research direction: perioperative organ protection and point of care ultrasound, email: zhengxinxin008@163.com.

Co-corresponding author: Zhao Lin yan, male,42 years old, associate chief physician, research direction: perioperative liver protection, email:zhaolinyanmail@126.com

\begin{abstract}
:
Objective: To study the cardiopulmonary changes in patients in post-anesthesia care unit by using BLUE protocol and FATE protocol. Methods: The cardiopulmonary ultrasonography of 60 patients with hypoxemia in post anesthesia care unit were analyzed retrospectively, and the
\end{abstract}


cardiopulmonary ultrasonography of patients with hypoxemia in different operations was compared. Results: A certain degree of pulmonary edema was found in patients with hypoxemia, which was mainly B7 line in patients with general surgery, B3 line in patients with thoracic surgery, and B3 line and debris sign in patients with spinal surgery. Cardiac ultrasound found that the inferior vena cava was larger than $2 \mathrm{~cm}$ in most patients, and the mutation rate of inferior vena cava was less than 25\%. Conclusion: Combined with cardiopulmonary ultrasonography can find abnormal pulmonary signs in patients. The decrease of oxygenation index after surgery is related to the surgical site and the tendency of fluid overload should be paid attention to.

Keywords: ultrasound, postoperative surgery, hypoxemia, pulmonary edema, inferior vena cava

Postoperative patients were difficult to be transferred to $\mathrm{CT}$ examination due to the presence of certain hemodynamic instability, residual sedatives and muscle relaxants, hypoxemia. The diagnostic rate of bedside lung ultrasound for severe pulmonary diseases such as pneumothorax, pulmonary edema and pleural effusion is similar to that of CT. In perioperative patients, due to inflammatory reaction, fluid overload, atelectasis and other reasons, the proportion of postoperative hypoxemia is relatively high, and even respiratory failure occurs in severe cases [1,2]. Pulmonary edema, atelectasis and pleural effusion are the main causes of perioperative hypoxemia, and bedside lung ultrasound can accurately identify the above causes after excluding cardiac factors and provide visual evidence during perioperative period, which is an important tool during perioperative period.

\section{Materials and Methods}

\section{Subjects}

The patients admitted to the post-anethesia care unit of the Second Affiliated Hospital of 
Dalian Medical University from September 2019 to February 2021 were retrospectively analyzed. 95\% and Ventilator SpO2 \&1t; 98\% is the entry criteria. Exclusion criteria: age \&lt; 18 years old; Severe hemodynamic disturbance requiring vasoactive drug support; Patients with large amount of subcutaneous emphysema after laparoscopic surgery who cannot be performed pulmonary ultrasound examination.

\section{Instruments and methods}

Use a Philips SPARQ color ultrasound meter with a convex array probe.

Blue protocol: The operator compared the size of the patient's hands together and placed them on the anterior chest wall, while the left hypothenar was placed on the subclavian edge. Check the position, blue spot: left hand three or four metacarpophalangeal joints; Lower blue point: right hand palm; Diaphragm point: Flat the right hypothenar; Plaps point: The point where the lower blue point intersects vertically and backward at the posterior axillary line.

Pulmonary ultrasound image line A: parallel to the pleural line, equidistant, repeated and gradually attenuated echo line; B3 line: it appears from the pleural line, points to the distal end of the screen and does not attenuate radium rays, and moves along with breathing. The distance between adjacent echo lines is $3 \mathrm{~mm}$. B7 line: property is the same as B3 line, adjacent echo line spacing is $7 \mathrm{~mm}$; Fragmentation sign: Solid - like tissue with a fragmented, irregular strong echo at its depth and near the boundary of the pneumonic lungs.

FATE protocol: The long parabernal axis, short parabernal axis, apical four-chambered heart and subxiphoid four-chambered heart (including inferior vena cava) sections were selected to observe the cardiac movement. $\mathrm{EF}$, diameter of inferior vena cava and variation rate of inferior vena cava were measured. 


\section{Result}

\section{General Information}

Fifty patients, aged 45 to 85 years, were included in the study, including 29 males and 21 females. There were 21 cases of general surgery, 11 cases of spinal surgery and 18 cases of thoracic surgery. Anesthesia recovery room extubation patients nasal catheter oxygen, oxygen concentration of $40 \%$; The oxygen concentration of patients on mechanical ventilation was $50 \%$.

\section{The occurrence of different pulmonary ultrasound images in each position}

In all patients, the most common occurrence of line A, which represents basically normal, is in the upper blue dot (50\%), followed by the lower blue point $(30 \%)$; The B3 line was most common at the diaphragmatic point (45\%), followed by the PLAPS point $(30 \%)$. The most common occurrence of the B7 line was at the PLAPS point $(60 \%)$, followed by the diaphragmatic point (20\%). Fragments were most common at PLAPS points $(50 \%)$, followed by lower blue dots $(25 \%)$.

\section{Ultrasound images of different surgical lung sites}

In general surgery patients, B7 line was the most common (70\%), followed by B3 line (20\%) and fragmentation sign (10\%). The patients in thoracic surgery were mainly B3 line $(60 \%)$, followed by B7 line (30\%) and debris sign (7\%). The majority of patients in spinal surgery were B3 line (50\%), followed by B7 line (20\%) and fragmentation sign (10\%).

Table1 imagine of lung ultrasound in patients after surgery

\begin{tabular}{|c|c|c|c|c|c|c|c|c|c|c|}
\hline & & $\begin{array}{c}\text { Left } \\
\text { upp } \\
\text { classif } \\
\text { y }\end{array}$ & $\begin{array}{c}\text { Left } \\
\text { low } \\
\text { er }\end{array}$ & $\begin{array}{c}\text { Left } \\
\text { er } \\
\text { PLAP } \\
\text { blue } \\
\text { poin } \\
\text { poin } \\
\mathrm{t}\end{array}$ & $\begin{array}{c}\text { S } \\
\text { point }\end{array}$ & $\begin{array}{c}\text { Left } \\
\text { diaphragma } \\
\text { tic point }\end{array}$ & $\begin{array}{c}\text { Rig } \\
\text { ht } \\
\text { er } \\
\text { blue } \\
\text { poin } \\
\mathrm{t}\end{array}$ & $\begin{array}{c}\text { Rig } \\
\text { ht } \\
\text { low } \\
\text { er } \\
\text { blue } \\
\text { poin } \\
\mathrm{t}\end{array}$ & $\begin{array}{c}\text { Right } \\
\text { PLAP } \\
\text { point }\end{array}$ & $\begin{array}{c}\text { Right } \\
\text { diaphragma } \\
\text { tic point }\end{array}$ \\
\hline $\begin{array}{c}\text { Thorac } \\
\text { ic } \\
\text { surgery }\end{array}$ & 33 & A & 10 & 3 & 4 & 1 & 12 & 1 & 2 & 2 \\
\hline
\end{tabular}




\begin{tabular}{|c|c|c|c|c|c|c|c|c|c|c|}
\hline & & B3 & 0 & 20 & 18 & 21 & 0 & 19 & 21 & 21 \\
\hline & & B7 & 23 & 10 & 10 & 11 & 18 & 12 & 10 & 9 \\
\hline & & $\begin{array}{l}\text { fragmentati } \\
\text { on sign }\end{array}$ & 0 & 0 & 1 & 0 & 3 & 1 & 0 & 1 \\
\hline \multirow{5}{*}{$\begin{array}{l}\text { Genera } \\
1 \\
\text { surgery }\end{array}$} & & & & & & & & & & \\
\hline & 15 & A & 14 & 2 & 0 & 1 & 12 & 3 & 1 & 1 \\
\hline & & B3 & 1 & 3 & 2 & 3 & 0 & 4 & 2 & 3 \\
\hline & & B7 & 0 & 10 & 13 & 10 & 3 & 7 & 10 & 10 \\
\hline & & $\begin{array}{l}\text { fragmentati } \\
\text { on sign }\end{array}$ & 0 & 0 & 2 & 1 & 0 & 1 & 2 & 1 \\
\hline \multirow{4}{*}{$\begin{array}{l}\text { Spinal } \\
\text { surgery }\end{array}$} & 12 & A & 8 & 2 & 1 & 1 & 6 & 1 & 0 & 1 \\
\hline & & B3 & 0 & 7 & 6 & 5 & 3 & 4 & 8 & 8 \\
\hline & & B7 & 4 & 2 & 3 & 4 & 3 & 5 & 4 & 3 \\
\hline & & $\begin{array}{l}\text { fragmentati } \\
\text { on sign }\end{array}$ & 0 & 1 & 2 & 2 & 0 & 2 & 0 & 0 \\
\hline
\end{tabular}

\section{Ultrasound images of lung with different oxygenation indexes}

The oxygenation index was mainly at the diaphragmatic point B7 in the $200-300$ group, and the oxygenation index was mainly at the PLAPS point B3 in the 100-200 group.

Table 2 Ultrasound images of lung with different oxygenation indexes

\begin{tabular}{|c|c|c|c|c|c|c|c|c|c|c|}
\hline & & $\begin{array}{c}\text { Left } \\
\text { upp } \\
\text { oxygenati } \\
\text { on index }\end{array}$ & $\begin{array}{c}\text { Left } \\
\text { low } \\
\text { er } \\
\text { er } \\
\text { blue } \\
\text { poin } \\
\text { t }\end{array}$ & $\begin{array}{c}\text { imagine } \\
\text { poin } \\
\mathrm{t}\end{array}$ & $\begin{array}{c}\text { Left } \\
\text { PLAP } \\
\text { S } \\
\text { point }\end{array}$ & $\begin{array}{c}\text { Left } \\
\text { diaphragm } \\
\text { atic } \\
\text { point }\end{array}$ & $\begin{array}{c}\text { Rig } \\
\text { ht } \\
\text { upp } \\
\text { er } \\
\text { blue } \\
\text { poin } \\
\text { t }\end{array}$ & $\begin{array}{c}\text { Rig } \\
\text { ht } \\
\text { low } \\
\text { er } \\
\text { blue } \\
\text { poin } \\
\text { t }\end{array}$ & $\begin{array}{c}\text { Right } \\
\text { PLAP } \\
\text { S } \\
\text { point }\end{array}$ & $\begin{array}{c}\text { Right } \\
\text { diaphragm } \\
\text { atic } \\
\text { point }\end{array}$ \\
\hline $200-300$ & 47 & A & 31 & 7 & 5 & 3 & 30 & 5 & 3 & 4 \\
\hline & & B3 & 1 & 28 & 15 & 25 & 3 & 26 & 29 & 31 \\
\hline
\end{tabular}




\begin{tabular}{|c|c|c|c|c|c|c|c|c|c|c|}
\hline & B7 & 24 & 21 & 26 & 24 & 21 & 23 & 23 & 21 \\
\hline $100-200$ & 3 & A & 1 & 0 & 0 & 0 & 0 & 0 & 0 & 0 \\
\hline & fragmentat & 0 & 1 & 5 & 3 & 3 & 3 & 2 & 1 \\
\hline & B3 sign & 0 & 2 & 3 & 2 & 0 & 1 & 2 & 1 \\
\hline & B7 & 3 & 1 & 0 & 1 & 3 & 1 & 1 & 1 \\
\hline
\end{tabular}

\section{Postoperative cardiac ultrasound characteristics of different patients}

Determination of EF value, EF\&gt; Left ventricular function was considered normal in $50 \%$, and EF\&GT in all patients. $50 \%$ is $96 \%$, $40 \% \& 1 t$; EF\&lt; $50 \%$ is $2 \%$, EF\&lt; $40 \%$ was $2 \%$. Patients with abnormal left heart function had completed cardiac ultrasound examination before surgery. Inferior vena cava (IVC) diameter of $2-2.5 \mathrm{~cm}$ was the most common $(85 \%)$, followed by $2.5-3 \mathrm{~cm}(15 \%)$. Inferior vena cava variation rate of spontaneous breathing \&lt; $35 \%$, the swelling rate of the inferior vena cava under mechanical ventilation. $10 \%$.

\section{Discuss}

Point of care ultrasound has been used in perioperative period for more than 30 years, and different procedures have been applied to critically ill patients to improve their prognosis [3-5]. At present, relevant training and practice have been carried out in critical medicine, emergency medicine, internal medicine and pediatrics, which can significantly improve the prognosis of patients and also play an important role in COVID-19 [6-8]. Blue process for dyspnea, FATE process for cardiac function assessment, and RUSH process for shock patients have been widely used in critical care medicine $[9,10]$. Postoperative hypoxemia was found in all patients, and a decrease in aerobic index was found by arterial blood gas analysis, but the etiology could not be accurately determined. Bedside ultrasound can detect common causes of 
abnormal oxygenation index of patients through visual evidence, such as pulmonary edema, pulmonary consolidation and pleural effusion, so as to guide the precise treatment of patients after surgery [11].

Lung is the only organ that receives total cardiac displacement, and postoperative pulmonary ultrasound changes are different in patients with different surgical sites, suggesting different postoperative effects on lung function. Meanwhile, all patients with general anesthesia are accompanied by a certain degree of atelectasis during mechanical ventilation, and the atelectasis is sustained due to delayed postoperative pulmonary expansion. In patients with general surgery, especially emergency patients, the release of inflammatory mediators in the surgical site into the lung tissue with circulation can induce acute lung injury, and then pulmonary edema appears. In patients with thoracic surgery, postoperative inflammatory reactions at the surgical site of the lung lead to the accumulation of neutrophils in the lung tissue and the release of inflammatory mediators, which are more prone to acute pulmonary edema. In patients with spinal surgery, postoperative release of inflammatory mediators, involvement of respiratory muscles in special sites such as cervical surgery, impaired cough ability and sputum excretion ability can lead to lung injury and even lung consolidation [11].

Perioperative fluid overload is also a major factor affecting lung function. In the past, perioperative fluid rehydration was mainly based on experience and lacked accurate indicators. Circulating capacity and fluid reactivity could be further determined by transthoracic and cardiac ultrasound examination with the help of relevant indicators of the inferior vena cava, and fluid overload could be determined by combining pulmonary ultrasound findings. According to previous studies, in patients with normal cardiac function, when the diameter of the inferior vena cava. $1.5 \mathrm{~cm}$ suggests insufficient circulation volume, accompanied by variation rate of autonomic breathing inferior vena cava $>50 \%$, swelling rate of inferior vena cava under mechanical ventilation $>15 \%$ indicates volume responsiveness in patients [2]. However, in this study, postoperative patients did not have volume responsiveness, suggesting that intraoperative fluid supplementation was sufficient and there was a tendency to aggravate pulmonary edema.

Previous studies have confirmed that pulmonary ultrasound can reflect the severity of 
pulmonary edema [10], and the same findings were also found in this study. With the decrease of oxygenation index, the number of patients with line B increased, and the incidence of line B3 was greater than that of line B7, indicating the aggravation of pulmonary edema.

Postoperative hypoxemia in patients is closely related to lung function injury, and previous studies have found three major causes, namely pulmonary edema, pulmonary consolidation and pleural effusion $[11,12]$. Is given priority to with pulmonary edema, and the study related to the study sample size is small, at the same time cardiopulmonary joint ultrasound can be ruled out further cardiac dysfunction caused by pulmonary edema, the circulating capacity can also be assessed, prompt perioperative application has a good bedside ultrasound in postoperative clinical significance, after system training specification can use visual evidence for the decision of perioperative [5], It has broad application prospect.

\section{Foundation item}

Teaching Reform Project of Dalian Medical University in 2019 (DYLX2019026)

\section{Reference}

[1] Yu Yuan, Shang You. Procedure of bedside ultrasound examination in severe critical patients. Chinese journal of diagnostics electronic, 2017,5 (3) :154-157

[2] Lyon M, Walton P, Bhalla V, Shiver SA. Ultrasound detection of the sliding lung sign by prehospital critical care providers. Am J Emerg Med. 2012; 30:48 5-8. Doi: 10.1016/ j.a jem. 2011.01.009.

[3] Wang Xiaoting, Liu Dawei, Yu Kaijiang, et al. Consensus of Severe ultrasound experts in China. Chinese journal of internal medicine, 2016,55 (11) :900-912.

[4] Cao Yu, Chao Yangong, Chen Libo, et al. Expert consensus on clinical application of bedside ultrasound in acute and critically ill patients. Chinese journal of emergency medicine, 2016,25 (1) :10-21.

[5] Moore CL,Copel JA. Point-of-care ultrasonography, N Engl JM,2011,364(8):749-757. 
10.1056/NEJMra0909487

[6] Yu Hong, Ze Peng, Yu Hai, et al. Perioperative Ultrasound: Essential Skills for anesthesiologists in the New Era, Journal of Chinese Clinical Anesthesiology, 2018,34(8):814-826.

[7] Daniel A. Lichtenstein.(2009)Lung Ultrasound in the Critically Ill.J Med Ultrasound, 17(3):125-142.

[8] Daniel A. Lichtenstein. BLUE-protocol and FALLS-protocol: two applications of lung ultrasound in the Critically Ill.CHEST,2015,147(6):1659-1670. DOI:10.1378/chest.14-1313

[9] ZHIXING ZHU, XIHUA LIAN, YIMING ZENG, WEIJING WU, ZHIRONG XU, YONGJIAN CHEN, JINGYUN LI, XIAOSHAN SU, LIQING ZENG, GUORONG LV. POINT-OF-CARE ULTRASOUND—A NEW OPTION FOR EARLY QUANTITATIVE ASSESSMENT OF PULMONARY EDEMA. Ultrasound in Medicine \& Biology ,2020, $46,(1): 1-10$

[10] Aron H. Ferreira, Antonio Pazin-Filho. (2020) Lung Ultrasound in a Patient With ARDS Secondary to Pancreatitis. CHEST,158(2): e85-e87.

[11] Hatem S A, Marcelo H M. (2020) The Sound of Silence -The Power of Lung Ultrasound in the Interstitial-Alveolar Syndrome. JACC: CASE REPORTS, 2(10):1550-1552.

[12] Matthieu Jabaudon, ules Audard, Bruno Pereira, Samir Jaber, Jean-Yves Lefrant, Raiko Blondonnet, Thomas Godet, Emmanuel Futier, Céline Lambert, MSc; Jean-Etienne Bazin,Julie A. Bastarache, Jean-Michel Constantin, Lorraine B. Ware. (2020) Early Changes Over Time in the Radiographic Assessment of Lung Edema Score Are Associated With Survival in ARDS. CHEST,158(6):2394-2403.

[13] Peng Qianyi, Zhang Lina, Li Li, et al. Analysis of pulmonary ultrasound image characteristics of postoperative intensive care patients based on Blue-plus protocol. Chinese journal of ultrasound imaging, 2017,26 (11) :976-981.

[14] D T Ashton- Cleary. Is thoracic ultrasound a viable alternative to conventional imaging in the critical care setting? B J A, 2013,111 (2) : 152-160.DOI:10.1093/bja/art076 